\title{
Postmortem distribution of MAB-CHMINACA in body fluids and solid tissues of a human cadaver
}

\author{
Koutaro Hasegawa $\cdot$ Amin Wurita $\cdot$ Kayoko Minakata $\cdot$ \\ Kunio Gonmori · Hideki Nozawa · Itaru Yamagishi · \\ Kanako Watanabe $\cdot$ Osamu Suzuki
}

Received: 15 February 2015 / Accepted: 23 February 2015/Published online: 10 March 2015

(C) Japanese Association of Forensic Toxicology and Springer Japan 2015

\begin{abstract}
During the latter part of 2014, we experienced an autopsy case in which 5-fluoro-ADB, one of the most dangerous synthetic cannabinoids, was identified and quantitated in solid tissues and in three herbal blend products [Forensic Toxicol (2015) 33:112-121]. At that time, although we suspected that there may be some drug(s) other than 5-fluoro-ADB in the herbal products, all trials to find it/them were unsuccessful. Subsequently, we carefully reexamined the presence of other synthetic cannabinoid(s) in the above herbal blend products using accurate mass spectrometry and found two new compounds, 5-fluoro-ADBPINACA and MAB-CHMINACA (Forensic Toxicol. doi: 10.1007/s 11419-015-0264-y). In the present communication, we report the distribution of MAB-CHMINACA in body fluids and solid tissue specimens collected from the same deceased individual (kept frozen at $-80{ }^{\circ} \mathrm{C}$ ) as described above for demonstration of 5-fluoro-ADB. Unexpectedly, unchanged MAB-CHMINACA could be identified and quantitated in whole blood and in pericardial fluid specimens, but it was below the detection limit $(0.1 \mathrm{ng} / \mathrm{ml})$ in the urine specimen. A higher concentration of MABCHMINACA could be found in all of the nine solid tissues; the highest concentration of MAB-CHMINACA was found in the liver (156 ng/g), followed by the kidney, pancreas and so on. The compounds were detected in all nine solid tissues; their levels were generally higher than those in the whole blood and pericardial fluid. Contrary to expectations, the
\end{abstract}

K. Hasegawa and A. Wurita contributed equally to this work.

K. Hasegawa ( $\square) \cdot$ A. Wurita $\cdot$ K. Minakata $\cdot$ K. Gonmori ·

H. Nozawa · I. Yamagishi · K. Watanabe · O. Suzuki

Department of Legal Medicine, Hamamatsu University School

of Medicine, 1-20-1 Handayama, Higashi-ku,

Hamamatsu 431-3192, Japan

e-mail: 07484771@hama-med.ac.jp concentration of MAB-CHMINACA in the adipose tissue was relatively low. Our results show that the victim smoked one of the three herbal blend products containing both MABCHMINACA and 5-fluoro-ADB, resulting in the coexistence of both compounds. It should be concluded that 5-fluoro-ADB and MAB-CHMINACA synergically exerted their toxicities, leading to death after a short interval. The differences in the distribution of 5-fluoro-ADB and MABCHMINACA among the cadaver specimens were also discussed in view of the structures of both compounds. To our knowledge, this is the first report to demonstrate MABCHMINACA in biological/human specimens.

Keywords MAB-CHMINACA - Synthetic cannabinoid Postmortem distribution · Solid tissue $\cdot$ LC-MS-MS .

5-Fluoro-ADB

\section{Introduction}

In recent years, various types of synthetic cannabinoids [14] and cathinones [5-8] have become widely distributed, and are now causing social problems throughout many parts of the world. Nowadays, the newly emerging drugs seem to have become more potent than the previous ones, and sometimes cause deaths [9-12].

During the latter part of 2014, three herbal blend products, all of which were open, were brought to our laboratory for analysis, together with a cadaver. At the time of autopsy, the primary cause of death was considered to be 5-fluoro-ADB poisoning, as this compound was detected in the stomach contents and in five solid tissues; 5-fluoro-ADB was detected at a concentration of $49.2 \pm 2.46 \mathrm{mg} / \mathrm{g}$ in the "GM sapphire" product, one of the three herbal blends [13]. At that time, however, we noticed unknown peaks other than 5-fluoro- 
ADB in the gas chromatography-mass spectrometry (GCMS) chromatograms for extracts of the three herbal blend products. We consulted the Cayman Spectral Library [14], but it did not suggest any compounds. Shortly after the autopsy, we re-examined the herbal blend extracts for the presence of other drug(s) using matrix-assisted laser desorption ionization (MALDI) tandem quadrupole time-of flight (QTOF) mass spectrometry. The MALDI tandem QTOF mass spectra strongly suggested the presence of 5-fluoro-ADB-PINACA and MAB-CHMINACA without their reference standards. After obtaining the reference standards, we compared the mass spectra of the extracts of the herbal blend products with those of the reference standards using both GC-MS and liquid chromatography-tandem mass spectrometry (LC-MS-MS). The mass spectra of the herbal blend extracts coincided with those of the reference standards, disclosing the presence of 5-fluoro-ADB-PINACA in "AL 37" and "AP 31" and MABCHMINACA in "GM sapphire" [15]; we then quantitated the concentrations of both compounds in the herbal blend products using the standard addition method. The concentrations of 5-fluoro-ADB-PINACA were $19.4 \pm 0.55$ and $19.0 \pm 0.47 \mathrm{mg} / \mathrm{g}$ (mean \pm standard deviation of triplicate determinations) for the herbal product brands " $\mathrm{AL}$ 37" and "AP 31," respectively; that of MAB-CHMINACA was $133 \pm 4.5 \mathrm{mg} / \mathrm{g}$ for the "GM sapphire" blend product [15].

In this communication, we identify and quantitate MAB-CHMINACA in various human specimens collected during autopsy. Unfortunately, however, 5-fluoro-ADBPINACA was only detected in the stomach contents. To our knowledge, this is the first report to identify and quantitate the new synthetic cannabinoid MAB-CHMINACA from biological specimens.

\section{Case history}

Human specimens were collected during the latter part of 2014 from the same deceased individual as described for demonstration of 5-fluoro-ADB [13]; specimens were stored at $-80{ }^{\circ} \mathrm{C}$. The deceased was a 30 -year-old man found in his room. Three opened, silver-colored herbal blend packages with brand names "AL 37," "AP 31" and "GM sapphire" were found nearby. Additional details of the case history are described in our previous report [13].

\section{Materials and methods}

\section{Materials}

The reference standards MAB-CHMINACA [synonym: ADB-CHMINACA, $N$-(1-amino-3,3-dimethyl-1-oxobutan- 2-yl)-1-(cyclohexylmethyl)-1 $H$-indazole-3-carboxamide], 5-fluoro-ADB-PINACA, AB-CHMINACA (internal standard, IS), and 5-fluoro-AB-PINACA (IS) were purchased from Cayman Chemical (Ann Arbor, MI, USA). All chemical structures of the above four synthetic cannabinoids are described in our previous report [15]. Other common chemicals used were of the highest purity commercially available. Plastic centrifuge tubes with caps (5-ml capacity, $6 \times 1.5 \mathrm{~cm}$ external diameter) and stainless beads $(5 \mathrm{~mm}$ external diameter) for crushing solid tissues were purchased from TAITEC, Saitama, Japan. QuEChERS dispersive-SPE centrifuge tubes with caps (2-ml capacity), each of which contained $25 \mathrm{mg}$ of primary secondary amine (PSA), $25 \mathrm{mg}$ of end-capped octadecylsilane $\left(\mathrm{C}_{18 \mathrm{EC}}\right)$, and $150 \mathrm{mg}$ of magnesium sulfate, and Captiva ND Lipids cartridges (3-ml capacity) were purchased from Agilent (Santa Clara, CA, USA).

Whole blood specimens from the right and left atria and femoral vein, pericardial fluid, urine, stomach contents, and nine solid tissue specimens (brain, lung, heart muscle, liver, spleen, kidney, pancreas, skeletal muscle and adipose tissue) were collected from the deceased at autopsy and kept frozen at $-80{ }^{\circ} \mathrm{C}$ until analysis; the adipose tissue specimen was obtained from the abdominal subcutaneous area.

Extraction procedure for human specimens

For analysis of MAB-CHMINACA, AB-CHMINACA was used as the IS; for that of 5-fluoro-ADB-PINACA, 5-fluoro-AB-PINACA was used. The extraction procedure included crushing each specimen with a bead beater-type homogenizer, QuEChERS dispersive solid-phase extraction, and filtration through a Captiva ND lipids cartridge. Among the solid tissues, some modified steps were added to the regular extraction procedure for the adipose tissue specimen, the details of which are described in our previous report [13].

\section{LC-MS-MS conditions}

LC-MS-MS conditions were exactly the same as those described in the previous report [13] except that ion transitions were $\mathrm{m} / \mathrm{z} 371 \rightarrow 241$ for MAB-CHMINACA, $\mathrm{m} / \mathrm{z}$ $357 \rightarrow 241$ for AB-CHMINACA (IS), $m / z 363 \rightarrow 318$ for 5-fluoro-ADB-PINACA, and $\mathrm{m} / z, 349 \rightarrow 304$ for 5-fluoroAB-PINACA (IS); the fragmentor voltage and collision energy were 120 and $25 \mathrm{~V}$ for MAB-CHMINACA, 120 and $21 \mathrm{~V}$ for AB-CHMINACA, 120 and $9 \mathrm{~V}$ for 5-fluoroADB-PINACA, and 120 and $9 \mathrm{~V}$ for 5-fluoro-ABPINACA, respectively. 
Standard addition method

The standard addition method is frequently used for analysis by atomic absorption spectroscopy [16]. Because this method does not require a blank matrix and completely overcomes matrix effects, we used it to analyse compound(s) in human specimens with quite different properties [11-13, 17-20]. Method principles and examples of the standard addition calibration curve were discussed in detail in our previous report [21].

\section{Matrix effects and recovery rates}

In our previous report [13] for analysis of 5-fluoro-ADB in human specimens, we used the heart muscle and adipose tissue as example matrices to demonstrate the matrix effects and recovery rates; in the present study, we used femoral vein blood and the liver as example matrices for analysis of MAB-CHMINACA. Other conditions of this experiment and the calculation method were the same as described previously [13].

\section{Results and discussion}

Identification trial for 5-fluoro-ADB-PINACA and MAB-CHMINACA

The extracts from all specimens were subjected to detection of peaks of 5-fluoro-ADB-PINACA by LC-MSMS in various detection modes. The protonated molecular ion at $\mathrm{m} / \mathrm{z}, 363$ could not be detected in any specimens of the deceased. However, in the selected reaction monitoring (SRM) mode, a very small peak at $\mathrm{m} / \mathrm{z} 318$ [above the limit of detection (LOD) and the below the limit of quantitation (LOQ)] could be detected only for the stomach contents; for all other specimens, any peak at $m / z, 318$ was below the LOD in the SRM mode.

In contrast to 5-fluoro-ADB-PINACA in the specimens, the concentrations of MAB-CHMINACA were found at much higher concentrations than those of 5-fluoro-ADBPINACA. It was possible to obtain product ion mass spectra from femoral vein whole blood and liver tissue specimens. Figure 1 shows an example of product ion mass spectra obtained from the extracts of femoral vein whole blood and liver tissue in comparison with that of the reference standard MAB-CHMINACA. The spectra obtained from the two extracts coincided with that of the reference standard MAB-CHMINACA, with no impurity peaks, confirming the detected compound was MABCHMINACA.
Validation of the method

Figure 2 shows an example of the SRM chromatograms for the target compound and the IS extracted from femoral vein whole blood and liver tissue. The target compound MAB-CHMINACA and IS AB-CHMINACA appeared at retention times of 8.72 and $7.93 \mathrm{~min}$, respectively. The bottom panel of Fig. 2 shows the absence of AB-CHMINACA in the liver tissue extract, though a very small peak was detected at the same retention time as that of $\mathrm{AB}$ CHMINACA with 100-fold magnification of the vertical axis. It seems reasonable to regard the small peak as a carryover of the AB-CHMINACA spiked into the liver tissue just before the run without the spiking of the IS. It should be noted that the backgrounds were generally very low, and there were no impurity peaks interfering with the target or IS peaks.

Table 1 shows the standard addition calibration equations for AMB-CHMINACA in 14 specimens, except for urine, in which the target compound was below the LOD. The correlation coefficients for all specimens were greater than 0.999. The LOD (signal-to-noise ratio $\geq 3$ ) for the compound using the present method was around $0.1 \mathrm{ng} / \mathrm{ml}$ or $\mathrm{g}$. The LOQ (signal-to-noise ratio $\geq 10$ ) was around $0.5 \mathrm{ng} / \mathrm{ml}$ or $\mathrm{g}$.

Because we employed the standard addition method for quantitation, without the use of blank specimens, it was impossible to present the usual accuracy and precision data. Instead, as shown in Table 2, we repeated intraday and interday determinations of MAB-CHMINACA in the femoral vein whole blood and liver tissue specimens as an example. The repeatability, expressed as relative standard deviations, was not greater than $14.8 \%$.

Although the standard addition method can overcome matrix effects and low recovery rates, we checked them under the present extraction conditions. In this study, we used acetonitrile deproteinization plus QuEChERS dispersive solid-phase extraction plus filtration through a Captiva ND Lipids cartridge coupled to an LC-MS-MS system. The matrix effects for MAB-CHMINACA were $76.9 \pm 1.70$ and $69.4 \pm 2.17 \%$ ( $n=3$ in each) for the femoral vein whole blood and liver tissue specimens, respectively. The recovery rates of the test compound calculated (for calculation method, see Ref. [13]]) were excellent at $88.8 \pm 11.2$ and $109 \pm 5.82 \% \quad(n=3$ in each) for the femoral vein whole blood and liver tissue specimens, respectively.

Postmortem distribution of MAB-CHMINACA in body fluids and solid tissues of the deceased

Table 3 shows the postmortem distribution of MABCHMINACA in body fluids, stomach contents, and nine 

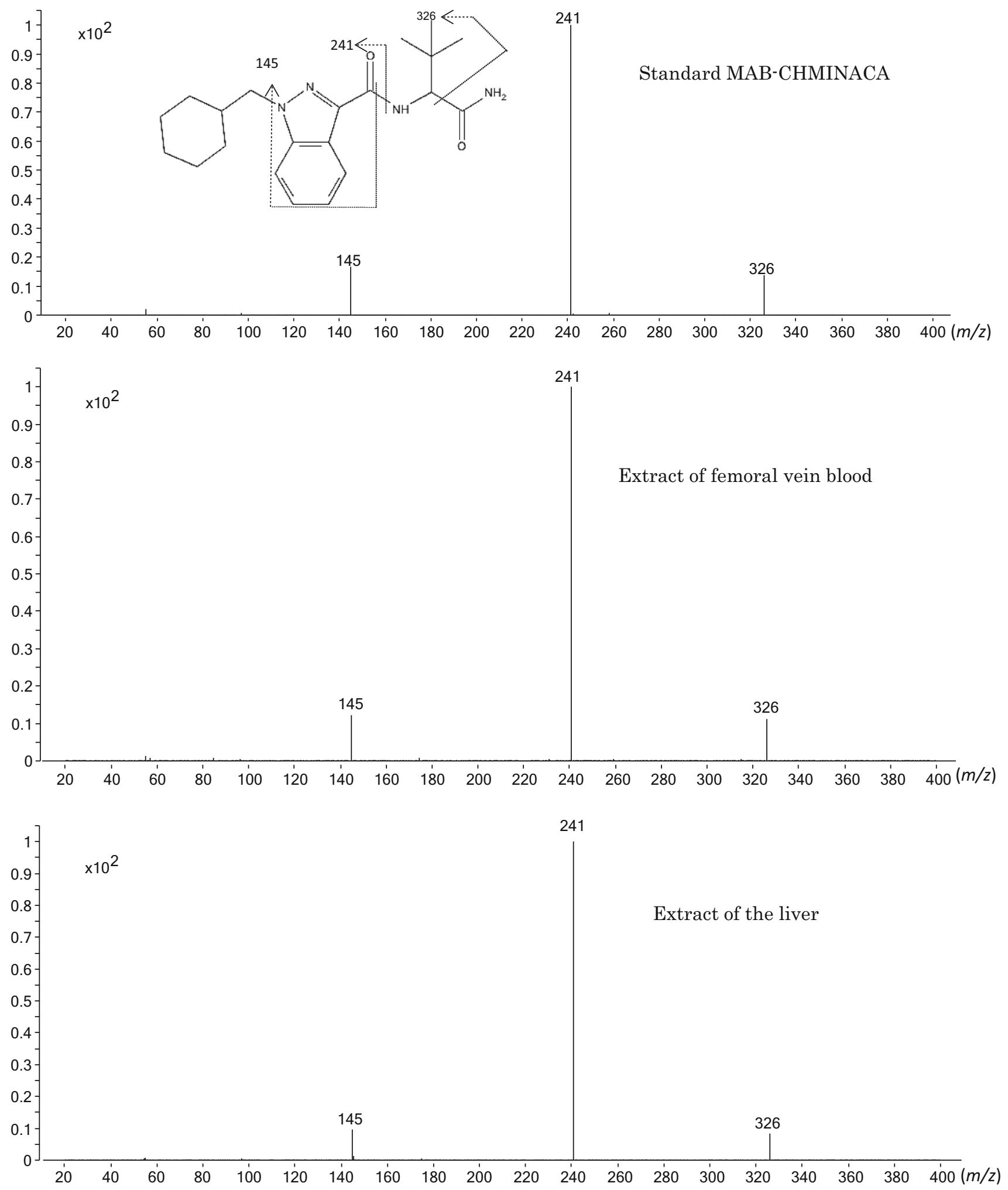

Fig. 1 Product ion mass spectra of the extracts of femoral vein whole blood and liver tissue specimens collected from the deceased in recorded by liquid chromatography-tandem mass spectrometry (LCcomparison with that of the reference standard MAB-CHMINACA, 

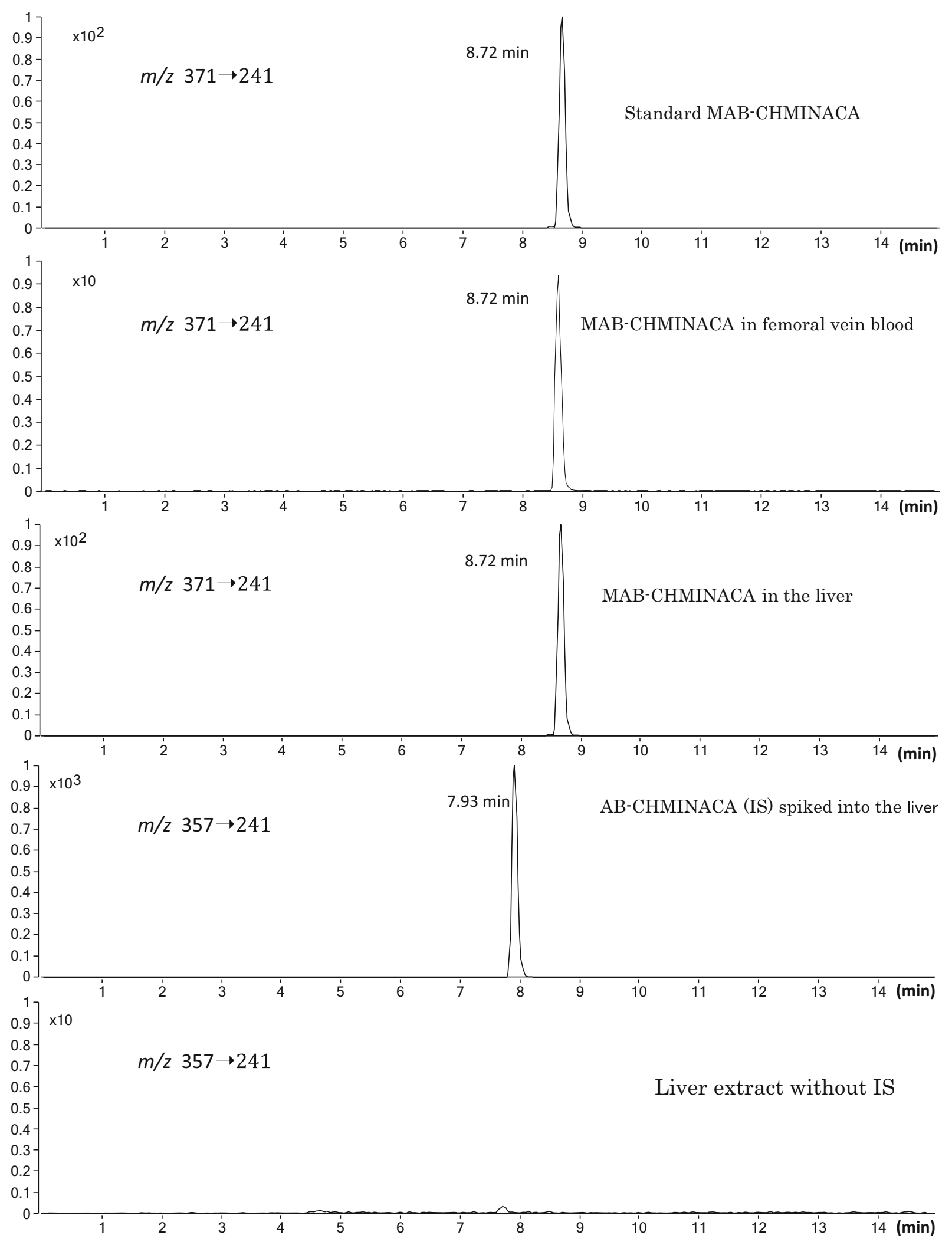

Fig. 2 Selected reaction monitoring chromatograms using LC-MSMS for the reference standard MAB-CHMINACA, the extracts of the femoral vein whole blood and liver tissue specimens, the internal standard (IS) AB-CHMINACA spiked into the liver tissue, and the extract of the liver tissue without addition of the IS 
Table 1 Standard addition calibration equations for MABCHMINACA in body fluids and solid tissues of the deceased
${ }^{a}$ If $y$ equals 0 , the pre-existing concentration $(x)$ can be calculated as a minus value

\begin{tabular}{|c|c|c|}
\hline Specimen & Equation $^{\mathrm{a}}$ & $\begin{array}{l}\text { Correlation } \\
\text { coefficient }(r)\end{array}$ \\
\hline Femoral vein blood & $y=0.001353 x+0.008194$ & 0.9999 \\
\hline Right heart blood & $y=0.001359 x+0.01447$ & 0.9999 \\
\hline Left heart blood & $y=0.001288 x+0.01199$ & 0.9999 \\
\hline Pericardial fluid & $y=0.001259 x+0.01882$ & 0.9995 \\
\hline Stomach contents & $y=0.001287 x+0.01358$ & 0.9998 \\
\hline Brain & $y=0.001017 x+0.01995$ & 0.9999 \\
\hline Lung & $y=0.001075 x+0.02416$ & 0.9998 \\
\hline Heart muscle & $y=0.001224 x+0.03390$ & 0.9999 \\
\hline Liver & $y=0.0009081 x+0.1414$ & 0.9999 \\
\hline Spleen & $y=0.00100 x+0.01725$ & 0.9998 \\
\hline Kidney & $y=0.0009232 x+0.03637$ & 0.9998 \\
\hline Pancreas & $y=0.001081 x+0.03447$ & 0.9999 \\
\hline Skeletal muscle & $y=0.001002 x+0.009822$ & 0.9999 \\
\hline Adipose tissue & $y=0.000246 x+0.004183$ & 0.9998 \\
\hline
\end{tabular}

Table 2 Examples of intraday and interday repeatability for determination of MAB-CHMINACA in postmortem femoral vein blood and in the liver of the deceased

\begin{tabular}{llllll}
\hline Specimen & \multicolumn{2}{l}{ Intraday $(n=5)$} & & \multicolumn{2}{l}{ Interday $(n=5)$} \\
\cline { 2 - 3 } & Concentration found $(\mathrm{ng} / \mathrm{ml})^{\mathrm{a}}$ & Repeatability $(\% \mathrm{RSD})$ & & Concentration found (ng/ml) & Repeatability $(\% \mathrm{RSD})$ \\
\hline Femoral vein blood & $6.05 \pm 0.185$ & 3.05 & $6.48 \pm 0.363$ & 5.60 \\
Liver & $156 \pm 5.83$ & 3.74 & $166 \pm 24.5$ & 14.8 \\
\hline
\end{tabular}

$R S D$ relative standard deviation

${ }^{\text {a }}$ Data given as mean \pm standard deviation (SD)

solid tissues. The concentration of the compound in urine was below the LOD (about $0.1 \mathrm{ng} / \mathrm{ml}$ ), despite detection of the compound in whole blood specimens. This suggests that the interval between inhalation of MAB-CHMINACA and cardiac arrest was so short that the compound could not reach the urinary bladder via the kidney and ureter. In the previous studies, synthetic cannabinoids were reported to accumulate in the adipose tissue at higher concentrations $[9,13,22]$. However, for MAB-CHMINACA in the present case, its concentration in the adipose tissue was not high, but was second from the lowest among the nine solid tissue specimens. This phenomenon may be also due to the shortness of interval between inhalation of the compound and cardiac arrest; it seems likely that it takes some time for MAB-CHMINACA to accumulate up to relatively high levels in the fat of the adipose tissue. The concentration of MAB-CHMINACA was outstandingly highest in the liver, followed by the kidney, pancreas and heart muscle.

According to our previous study [13], it is evident that this victim smoked the herbal blend of "GM sapphire." In another study [15], we also disclosed a high concentration of MAB-CHMINACA from the same herbal blend item
"GM sapphire," and here we detected MAB-CHMINACA from body fluid and solid tissue specimens collected from the same cadaver as used in the previous study [13]. These results show that 5-fluoro-ADB and MAB-CHMINACA coexisted in the "GM sapphire" herbal blend that was smoked by the victim. It should be concluded, therefore, that 5-fluoro-ADB and MAB-CHMINACA synergically exerted their toxicities, leading to death after a short interval.

It seems useful to discuss the difference in distribution among the specimens collected from the same deceased for 5-fluoro-ADB and MAB-CHMINACA. In addition, the contents of both compounds in "GM sapphire" are known; they were $49.2 \pm 2.46$ [13] and $133 \pm 4.5 \mathrm{mg} / \mathrm{g}$ [15], respectively. The first point to be mentioned is the much higher concentrations of MAB-CHMINACA than those of 5-fluoro-ADB in the specimens; the concentrations of 5-fluoro-ADB were only 3.18, 1.90, 1.82, 1.17, 1.61 and $7.95 \mathrm{ng} / \mathrm{g}$ in stomach contents, the brain, heart muscle, spleen, pancreas and adipose tissue, respectively, and were below the LOD for body fluid specimens; very small peaks appeared for the lung, liver, kidney, and 
Table 3 Concentrations of MAB-CHMINACA in body fluids and solid tissues of the deceased

\begin{tabular}{ll}
\hline Specimen & Concentration $(\mathrm{ng} / \mathrm{ml}$ or g) \\
\hline Femoral vein blood & $6.05 \pm 0.185$ \\
Right heart blood & $10.6 \pm 0.667$ \\
Left heart blood & $9.30 \pm 0.334$ \\
Pericardial fluid & $14.9 \pm 0.768$ \\
Stomach contents & $10.6 \pm 0.133$ \\
Urine & $\mathrm{ND}$ \\
Brain & $19.6 \pm 0.572$ \\
Lung & $22.5 \pm 1.29$ \\
Heart muscle & $27.7 \pm 0.933$ \\
Liver & $156 \pm 5.83$ \\
Spleen & $17.2 \pm 1.00$ \\
Kidney & $39.4 \pm 1.69$ \\
Pancreas & $31.9 \pm 0.415$ \\
Skeletal muscle & $9.80 \pm 0.28$ \\
Adipose tissue & $17.0 \pm 1.44$ \\
\hline
\end{tabular}

Data given as mean \pm SD obtained by triplicate determinations

$N D$ not detectable [lower than the detection limit $(0.1 \mathrm{ng} / \mathrm{ml})$ ]

skeletal muscle, but they were below the LOQ [13]. Even if we take into consideration the fact that the concentration of MAB-CHMINACA is 2.7-fold higher than that of 5 -fluoro-ADB in the herbal blend product "GM sapphire," the concentrations of MAB-CHMINACA in various human specimens are much higher than the values expected (Table 3) when compared with those of 5-fluoro-ADB [13]. In addition, the concentrations of MAB-CHMINACA were $6.05-10.6 \mathrm{ng} / \mathrm{ml}$ in whole blood specimens, while those of 5-fluoro-ADB in whole blood were below the LOD. These differences in concentrations between the two compounds may be largely due to the stability of the compounds; MAB-CHMINACA has a primary amino group at the terminal part and a cyclohexylmethyl moiety attached to the nitrogen, both of which are much more stable and resistant to chemical decomposition and/or metabolism than the methoxy group and the 5-fluoropentyl moiety present in the 5-fluoroADB structure in an alive/dead human body.

Although the outstandingly high concentration of MABCHMINACA in the liver is difficult to explain, the secondhighest concentration of the compound in the kidney (Table 3) may be due to the ability of this compound to excrete into urine via the kidney, while the concentration of 5 -fluoro-ADB in the kidney was below the LOQ [13]. This contrast seems to be due to the hydrophilic nature of the compounds; MAB-CHMINACA is more hydrophilic due to the presence of a primary amino group than is 5-fluoroADB with the methoxy group.

\section{Conclusions}

In the present study, we identified and quantitated MABCHMINACA in human specimens collected from the same victim as used in the previous study on the demonstration of 5-fluoro-ADB. The concentration of MAB-CHMINACA was outstandingly highest in the liver, followed by the kidney, pancreas and heart muscle. The compound was also detected in whole blood and pericardial fluid, but not in urine. To our knowledge, this is the first report to demonstrate MAB-CHMINACA from biological/human specimens.

Conflict of interest There are no financial or other relations that could lead to a conflict of interest.

\section{References}

1. Zuba D, Byrska B (2013) Analysis of the prevalence and coexistence of synthetic cannabinoids in "herbal high" products in Poland. Forensic Toxicol 31:21-30

2. Kikura-Hanajiri R, Uchiyama N, Kawamura M, Goda Y (2013) Changes in the prevalence of synthetic cannabinoids and cathinone derivatives in Japan until early 2012. Forensic Toxicol 31:44-53

3. Chung H, Choi H, Heo S, Kim E, Lee J (2014) Synthetic cannabinoids abused in South Korea : drug identifications by the National Forensic Service from 2009 to June 2013. Forensic Toxicol 32:82-88

4. Uchiyama N, Shimokawa Y, Kawamura M, Kikura-Hanajiri R, Hakamatsuka $T$ (2014) Chemical analysis of a benzofuran derivative, 2-(2-ethylaminopropyl)benzofuran (2-EAPB), eight synthetic cannabinoids, five cathinone derivatives, and five other designer drugs newly detected in illegal products. Forensic Toxicol 32:266-281

5. Shima N, Katagi M, Kamata H, Matsuta S, Nakanishi K, Zaitsu K, Kamata T, Nishioka H, Miki A, Tatsuno M, Sato T, Tsuchihashi H, Suzuki K (2013) Urinary excretion and metabolism of the newly encountered designer drug 3,4-dimethylmethcathinone in humans. Forensic Toxicol 31:101-112

6. Namera A, Konuma K, Kawamura M, Saito T, Nakamoto A, Yahata M, Ohta S, Miyazaki S, Shiraishi H, Nagao M (2014) Time-course profile of urinary excretion of intravenously administered $\alpha$-pyrrolidinovalerophenone and $\alpha$-pyrrolidinobutiophenone in a human. Forensic Toxicol 32:68-74

7. Zaitsu K, Katagi M, Tsuchihashi H, Ishii A (2014) Recently abused synthetic cathinones, $\alpha$-pyrrolidinophenone derivatives: a review of their pharmacology, acute toxicity, and metabolism. Forensic Toxicol 32:1-8

8. Miserez B, Ayrton O, Ramsey J (2014) Analysis of purity and cutting agents in street mephedrone samples from South Wales. Forensic Toxicol 32:305-310

9. Saito T, Namera A, Miura N, Ohta S, Miyazaki S, Osawa M, Inokuchi S (2013) A fatal case of MAM-2201 poisoning. Forensic Toxicol 31:333-337

10. Namera A, Urabe S, Saito T, Torikoshi-Hatano A, Shiraishi H, Arima Y, Nagao M (2013) A fatal case of 3,4-methylenedioxypyrovalerone poisoning: coexistence of $\alpha$-pyrrolidinobutiophenone and $\alpha$-pyrrolidinovalerophenone in blood and/or hair. Forensic Toxicol 31:338-343 
11. Hasegawa K, Suzuki O, Wurita A, Minakata K, Yamagishi I, Nozawa H, Gonmori K, Watanabe K (2014) Postmortem distribution of $\alpha$-pyrrolidinovalerophenone and its metabolite in body fluids and solid tissues in a fatal poisoning case measured by LCMS-MS with the standard addition method. Forensic Toxicol 32:225-234

12. Hasegawa K, Wurita A, Minakata K, Gonmori K, Nozawa H, Yamagishi I, Suzuki O, Watanabe K (2014) Identification and quantitation of a new cathinone designer drug PV9 in an "aroma liquid" product, antemortem whole blood and urine specimens, and a postmortem whole blood specimen in a fatal poisoning case. Forensic Toxicol 32:243-250

13. Hasegawa K, Wurita A, Minakata K, Gonmori K, Yamagishi I, Nozawa H, Watanabe K, Suzuki O (2015) Identification and quantitation of 5-fluoro-ADB, one of the most dangerous synthetic cannabinoids, in the stomach contents, and solid tissues of a human cadaver, and in some herbal products. Forensic Toxicol 33:112-121

14. Cayman Chemical (2014) Cayman spectral library. https://www. caymanchem.com/app/template/SpectralLibrary.vm. Accessed Oct 2014

15. Wurita A, Hasegawa K, Minakata K, Gonmori K, Yamagishi I, Nozawa H, Watanabe K, Suzuki O (2015) Identification and quantitation of 5-fluoro-ADB-PINACA and MAB-CHMINACA in dubious herbal products. Forensic Toxicol. doi:10.1007/ s11419-015-0264-y

16. Bonilla E (1978) Flameless atomic absorption spectrophotometric determination of manganese in rat brain and other tissues. Clin Chem 24:471-474

17. Wurita A, Hasegawa K, Minakata K, Watanabe K, Suzuki O (2014) A large amount of new designer drug diphenidine coexisting with a synthetic cannabinoid 5-fluoro-AB-PINACA found in a dubious herbal product. Forensic Toxicol 32:331-337

18. Wurita A, Suzuki O, Hasegawa K, Gonmori K, Minakata K, Yamagishi I, Nozawa H, Watanabe K (2013) Sensitive determination of ethylene glycol, propylene glycol and diethylene glycol in human whole blood by isotope dilution gas chromatographymass spectrometry, and the presence of appreciable amounts of the glycols in blood of healthy subjects. Forensic Toxicol $31: 272-280$

19. Wurita A, Suzuki O, Hasegawa K, Gonmori K, Minakata K, Yamagishi I, Nozawa H, Watanabe K (2014) Presence of appreciable amounts of ethylene glycol, propylene glycol, and diethylene glycol in human urine of healthy subjects. Forensic Toxicol 32:39-44

20. Wurita A, Suzuki O, Hasegawa K, Gonmori K, Minakata K, Yamagishi I, Nozawa H, Watanabe K (2014) Occurrence of postmortem production of ethylene glycol and propylene glycol in human specimens. Forensic Toxicol 32:162-168

21. Wurita A, Hasegawa K, Minakata K, Gonmori K, Nozawa H, Yamagishi I, Suzuki O, Watanabe K (2014) Postmortem distribution of $\alpha$-pyrrolidinobutiophenone in body fluids and solid tissues of a human cadaver. Leg Med 16:241-246

22. Hasegawa K, Wurita A, Minakata K, Gonmori K, Nozawa H, Yamagishi I, Watanabe K, Suzuki O (2015) Postmortem distribution of AB-CHMINACA, 5-fluoro-AMB and diphenidine in body fluids and solid tissues in a fatal poisoning case: usefulness of the adipose tissue for detection of the drugs in the unchanged forms. Forensic Toxicol 33:45-53 